\title{
Frecuencia y pertinencia del perfil lipídico como examen inicial en vértigo periférico
}

\author{
Lipid profile frequency and relevancy as an initial test for \\ peripheral vertigo
}

Isidro Torres-Castro ${ }^{1}$, Hassan Hendauss-Waked ${ }^{2}$, Antonio Baena-Rivero $^{3}$ y Carlos E. Granados-Gómez ${ }^{3}$

1 Colsanitas. Bogotá, Colombia. tocais@yahoo.com

2 Clínica Universitaria Teletón. Bogotá, Colombia. miraj3000@yahoo.com

3 Universidad de la Sabana. Bogotá,Colombia.antonio.baena@unisabana.edu.co, caregra@gmail.com

Recibido 26 Noviembre 2010/Enviado para Modificación 2 Octubre 2011/Aceptado 22 Octubre 2011

\section{RESUMEN}

Objetivos Determinar la prevalencia de la solicitud del perfil lipídico como ayuda diagnóstica de primera línea en pacientes con impresión clínica de vértigo periférico, así como un análisis de la relación costo-beneficio de dicha solicitud.

Métodos Estudio de tipo corte transversal retrospectivo. Revisión de 201 historias de pacientes atendidos en la consulta externa tanto de especialistas como de médicos generales de la Clínica Universitaria Teletón y Casa chía, con diagnóstico de vértigo periférico, en el periodo comprendido entre enero de 2005 y julio de 2008. Resultados Las edades de los pacientes oscilaron entre 6 y 87 años, el $36 \%$ de sexo masculino y el $64 \%$ femenino. El perfil lipídico fue solicitado como prueba diagnóstica inicial para el manejo de vértigo a un $76 \%$ de los pacientes. Los perfiles lipídicos hallados fueron normales en el $80 \%$ de los pacientes a quienes se les solicitó. Se presenta el análisis de la relación costo-beneficio de esta prueba. Conclusiones Existe una alta prevalencia de solicitud de perfil lipídico como estudio de primera línea en el abordaje de pacientes con vértigo periférico, aunque no existe evidencia que avale esta solicitud, se requiere retroalimentación al cuerpo médico tanto de atención primaria como especialistas.

Palabras Clave: Vértigo, lípido, prevalencia, análisis, uso diagnóstico (fuente: DeCS, BIREME).

\section{ABSTRACT}

Objective Determining the prevalence of requesting lipid profile as a first-line diagnostic method in patients having a clinical impression of peripheral vertigo and also determining such request's cost- benefit ratio. 
Methods This was a retrospective cross-sectional study of 201 clinical charts regarding patients diagnosed as having peripheral vertigo at the Teletón teaching hospital's outpatient services in Chía between January 2005 and July 2008. Clinical charts drawn up by both general practitioners and medical specialists at first-time visit were compiled and analyzed

Results The patients were aged 6 to 87 years old; $36 \%$ were males and $64 \%$ females. Lipid profile was requested for $76 \%$ of the patients as initial diagnostic method; it was found that $80 \%$ of the results revealed no abnormality.

Conclusion Although a high prevalence regarding a request for lipid profile first-line study was found for patients having peripheral vertigo, there was no scientific evidence supporting such requirement. Ordering this kind of study not only increases unnecessary costs concerning diagnostic methods but also involves unjustified treatment. Feedback is needed for both doctors and first attention staff to ensure that this type of practice becomes changed.

Key Words: Vertigo, lipid, prevalence, analysis, diagnostic use (source: $M e S H, N L M$ ).

$\mathrm{E}$ xiste controversia acerca de la relación entre dislipidemia y trastornos vertiginosos. La evidencia de no correlación entre dislipidemia y vértigo periférico es sustancialmente más numerosa que aquella que si muestra. Observaciones aisladas muestran que algunos médicos persisten solicitando análisis de lípidos como prueba de primera línea en el diagnóstico del vértigo periférico.

En Estados Unidos el $1 \%$ de las consultas generales son por vértigo, con una prevalencia de $23 \%$ e incidencia de $3 \%$ en la población general (1-3). Se considera que el $70 \%$ de los pacientes con vértigo son atendidos inicialmente por médicos generales, médicos internistas o médicos familiares y sólo $4 \%$ son remitidos a otros especialistas. (4) La prevalencia de esta patología y la solicitud del perfil lipídico hace que se aumenten los costos para el sistema de salud en estudios de laboratorio, que la evidencia establece como innecesarios. El costo de un perfil lipídico completo, en Colombia, oscila entre COP\$19.100 y COP\$20.910.

Estudios del programa Nacional de Educación de Colesterol (NCEP) en la detección, evaluación y tratamiento de niveles altos de colesterol sérico en su tercer reporte ATPIII (Adult Treatment Panel), muestran que las alteraciones del perfil lipídico generalmente son asintomáticas. Los signos ocasionalmente descritos son xantomas y lipomas. (5-6-7). El diagnóstico de alteraciones en los lípidos, en la mayoría de pacientes es realizado mediante pruebas de tamizaje o en análisis aleatorios que los médicos ordenan a sus pacientes. Hay estudios que recomiendan solicitud de perfil lipídico en 
pacientes con vértigo, pero son de difícil caracterización (8). Otros estudios relacionan las alteraciones de los lípidos con disfunción del aparato vestíbulococlear. Dicha alteración se relaciona con neuritis vestibular o tinnitus consideradas indicaciones para solicitar análisis de lípidos (9-13).

En un análisis alemán, multivariable, se encontró relación entre síntomas vertiginosos y enfermedad cardiovascular asociada a alteraciones de perfil lipídico (14). El doctor Colledge y colaboradores de la universidad de Edinburgo concluyen, luego de analizar los resultados de los exámenes de laboratorio solicitados en adultos mayores con vértigo que estos fueron normales y no distinguibles de un grupo control sin vértigo (15).

Reconocidos textos de medicina y publicaciones (16-20) enfocan el diagnóstico de vértigo a la parte clínica y no sugieren el perfil lipídico como examen inicial para el estudio de estos pacientes. Sin embargo algunos textos mencionan otros exámenes de laboratorio como glicemia, pruebas tiroideas, hemograma y velocidad de sedimentación globular, sin especificar la evidencia en la cual se sustenta esta solicitud. Por último, el estudio de Espinosa Sánchez y colaboradores en su libro "El paciente con vértigo en urgencias" también hacen énfasis en el diagnóstico clínico y no en pruebas de laboratorio para esta patología (21).

\section{MÉTODOS}

Tipo de estudio: Corte transversal retrospectivo.

Población de estudio: Pacientes atendidos por primera vez en la consulta externa de la Clínica Universitaria Teletón y Casa chía, con diagnóstico de vértigo periférico.

Criterios de inclusión:

- Pacientes atendidos en la consulta externa de la Clínica Universitaria Teletón y Casa Chía en el periodo comprendido entre enero 2005 y julio 2008 con diagnóstico clínico de: vértigo paroxístico benigno (Código CIE $10 \mathrm{H} 811$ ), otros vértigos periféricos (Código CIE 10 H813), vértigo epidémico (Código CIE 10 A881).

- Pacientes cuyas historias clínicas permitieran verificar diagnóstico de vértigo y definir solicitud de ayudas diagnósticas. 
Criterios de exclusión:

- Diagnóstico de vértigo central.

- Pacientes atendidos por medicina familiar; protocolos de manejo de vértigo de Medicina Familiar y Comunitaria de la Universidad de la Sabana proscriben solicitar perfil lipídico a pacientes con diagnóstico aislado de vértigo.

- Pacientes a quienes se les solicitó perfil lipídico.

Se revisaron las historias clínicas de todos los pacientes atendidos en Consulta Externa con diagnóstico CIE10: H811 vértigo paroxístico benigno, H813 otros vértigos periféricos, A881 vértigo epidémico, en el periodo comprendido entre enero 2005 y julio 2008 de la Clínica Universitaria Teletón y casa Chía, obteniéndose un total de 738 consultas. Se clasificaron los pacientes, eliminando aquellos que habían consultado más de una vez (350 historias) y tomando solo la primera consulta con estos diagnósticos, obteniéndose una población de 388 pacientes. Posteriormente se aplican criterios de inclusión y exclusión eliminándose 91 historias por diagnóstico errado de vértigo y a 96 de ellas no se obtuvieron acceso por el sistema. Se alcanzó una población final de 201 pacientes.

Medición de resultados y plan de análisis

La medición primaria incluyó la prevalencia de la solicitud de perfil lipídico en pacientes con vértigo periférico. Hubo medición secundaria al analizar datos de perfil lipídico y clasificar esto según niveles de normalidad. Análisis estadísticos utilizando funciones del programa Excel. Estadística descriptiva, usando cuantificación de frecuencias para variables nominales y ordinales y medidas de tendencia central y de dispersión para variables cuantitativas. No se pretende extrapolar a una población por lo cual no se realiza prueba de hipótesis.

\section{RESULTADOS}

De los 201 pacientes, el $36 \%$ corresponden al sexo masculino y el $64 \%$ al femenino, con un promedio de edad de $53 \pm 19$ años. El rango de edad estuvo comprendido entre 8 y 91 años y fueron clasificados de acuerdo al ciclo vital individual. El $31 \%$ de los pacientes mayores de 65 años, $35 \%$ edades entre 45 y 64 años, $31 \%$ oscilaban entre 20 y 44 años, $2,5 \%$ entre 10 y 19 años y $0,5 \%$ entre 6 y 9 años. 
El mayor porcentaje de pacientes $(44 \%)$ fue atendido por médicos especialistas en otorrinolaringología, seguido de un $25 \%$ por especialistas en neurología y un $24 \%$ por médicos generales. El $7 \%$ restante fue atendido por especialistas en neurocirugía (4\%) y medicina interna (3\%).

De los 201 pacientes estudiados, les fue solicitado perfil lipídico como prueba diagnóstica inicial en el manejo de vértigo, a 153 (76\%) (Tabla 1).

Tabla 1. Atención según profesional médico y solicitud de perfil lipídico

\begin{tabular}{lcc}
\hline $\begin{array}{c}\text { Atención por tipo de profesional } \\
\text { medico }\end{array}$ & $\begin{array}{c}\text { Pacientes } \\
\text { atendidos } \\
\mathrm{n}\end{array}$ & $\begin{array}{c}\text { Pacientes a quienes solicitaron } \\
\text { perfil lipídico } \\
\mathrm{n}(\%)\end{array}$ \\
\hline Especialista en otorrinolaringología & 89 & $67(75)$ \\
Especialistas en neurología. & 50 & $45(90)$ \\
Médico general & 47 & $32(68)$ \\
Especialista en neurocirugía & 8 & $3(38)$ \\
Especialista en Medicina interna & 7 & $6(85)$ \\
Totales & 201 & $153(76)$ \\
\hline
\end{tabular}

Descripción del perfil lipídico

De los 201 pacientes incluidos, les fue solicitado perfil lipídico o algunas de sus fracciones a 153 pacientes, de los cuales sólo se obtuvieron los resultados completos o parciales en 137 pacientes, lo que corresponde al $90 \%$.

Los valores del perfil lipídico hallados, se clasificaron en rangos de normalidad según lo descrito en el Programa Nacional de Educación de Colesterol (NCEP) en la detección, evaluación y tratamiento de niveles altos de colesterol sérico en su tercer reporte ATP III (Adult Treatment Panel) (6-7).

$\mathrm{Al}$ analizar los datos de este estudio se encontraron niveles de colesterol HDL dentro de valores normales en $80 \%$ de los perfiles hallados (Tabla 2).

Tabla 2. Clasificación de valores de HDL por rango

\begin{tabular}{ccc}
\hline $\mathrm{HDL}$ mg/dl & $\begin{array}{c}\text { Número de } \\
\text { pacientes }\end{array}$ & Porcentaje \\
\hline$\leq 39$ & 18 & 20 \\
$\geq 40$ & 71 & 80 \\
Total & 89 & 100 \\
\hline
\end{tabular}

Con los valores de triglicéridos se encontró un comportamiento similar obteniéndose un $86 \%$ con valores por debajo de $200 \mathrm{mg} / \mathrm{dl}$ (Tabla 3). 
Tabla 3. Clasificación de valores de triglicéridos por rango

\begin{tabular}{ccc}
\hline Triglicéridos $\mathrm{mg} / \mathrm{dl}$ & $\begin{array}{c}\text { Número de } \\
\text { pacientes }\end{array}$ & Porcentaje \\
\hline$\leq 199$ & 112 & 86 \\
$\geq 200$ & 18 & 14 \\
Total & 130 & 100 \\
\hline
\end{tabular}

Con respecto al colesterol LDL un valor por encima de $150 \mathrm{mg}$ se observó en un $25 \%$ de los perfiles lipídicos encontrados. Es importante aclarar que en este estudio no se tomó en cuenta la clasificación de riesgo cardiovascular del paciente para establecer las metas de colesterol LDL ya que se sale del objetivo principal del mismo (Tabla 4).

Tabla 4. Clasificación de valores de LDL por rango

\begin{tabular}{ccc}
\hline Valor LDL mg/dl & Número de Pacientes & Porcentaje \\
\hline$<100$ & 11 & 13 \\
De $100-130$ & 30 & 36 \\
De $130-150$ & 22 & 26 \\
$>150$ & 21 & 25 \\
Total & 84 & 100 \\
\hline
\end{tabular}

\section{DISCUSIÓN}

Existe una alta proporción de médicos generales y especialistas que solicitan el perfil lipídico como examen de primera línea en pacientes con diagnóstico de vértigo periférico aunque la evidencia no avale esta solicitud. Sin embargo, la mayoría de valores hallados de perfil lipídico estuvieron en su mayoría dentro de rangos aceptados como normales lo que apunta hacia la ausencia de relación entre dislipidemia y vértigo periférico.

Los costos generados por solicitud de paraclínicos innecesarios representan un aumento en gastos para el sistema de salud. En este estudio, donde la población fue pequeña, el costo no justificado por solicitud de perfil lipídico fue aproximadamente de COP\$ 3199 230. La solicitud del perfil lipídico en pacientes con vértigo periférico no sólo acarrea gastos por solicitud del paraclínico sino también en tratamientos farmacológicos no justificados, en aras del mejoramiento del vértigo periférico.

Estudios como el de Nicki y Robin (22) muestran la poca utilidad de la solicitud de paraclínicos tanto imagenologicos como de laboratorio en dicha patología. Otros estudios como Kazmierczak, Doroszewska (23) y Lin, Young (24) muestran que los perfiles lipídicos en los pacientes estudiados con vértigo 
no diferían de aquellos individuos sin dicha patología, lo que se confirma en el presente estudio. Por otra parte, los resultados de perfil lipídico agrupados por rangos de normalidad son similares a los de otros estudios encontrados $(2,8,25)$. Es importante recalcar que los textos de referencia de neurología, otorrinolaringología, y medicina familiar en sus capítulos de vértigo no mencionan el perfil lipídico como una prueba indicada en pacientes con vértigo periférico, así tampoco las guías de manejo de pacientes en urgencias con ese diagnostico.

Un aspecto importante de este estudio es la evaluación de costos innecesarios a los que se ve sometido el sistema de salud por solicitud de paraclínicos que no cambian el direccionamiento de manejo de una patología dada. Teniendo en cuenta la alta prevalencia tanto de vértigo periférico como de dislipidemia, se puede incurrir no sólo en aumento de costos en pruebas de laboratorio, sino también en manejos farmacológicos innecesarios por paradigmas médicos. Un estudio de calidad en salud realizado en Israel demostró que el $35 \%$ del presupuesto que se destinó a la salud en ese país se desperdició en costos no necesarios en pruebas de laboratorios y manejo farmacológico. Otro estudio de la universidad de Pittsburgh: "Healthcare Financial Management" refiere que hay más de 350 millones de dólares anualmente de costos innecesarios por exámenes de laboratorio que no cambian el estado de salud del paciente (26).

Pareciera existir una presión cultural, que puede estar sesgando el criterio médico y llevándolo a no basarse en la evidencia para solicitar paraclínicos no justificados tales como el perfil lipídico en caso de vértigo periférico.

Se recalca la importancia de este estudio para el control interno de instituciones evaluadas, la adopción de guías de manejo de las diferentes patologías en Consulta Externa, basadas en la evidencia para aumento de costo-efectividad y mejoramiento continuo de la calidad de atención y la implementación de programas de educación a médicos generales y especialistas

\section{REFERENCIAS}

1. Hanley K, O'Dowd T, Considine N. A systematic review of vertigo in primary care. Br J Gen Pract. 2001; 51:666-71.

2. Kroenke K, Lucas CA, Rosenberg ML, Scherokman B, Herbers JE, Jr, Wehrle PA, et al. Causes of persistent dizziness. A prospective study of 100 patients in ambulatory care. Ann Intern Med. 1992; 117:898-904. 
3. Neuhauser HK, Radtke A, von Brevern M, Lezius F, Feldmann M, Lempert T, Burden of Dizziness and Vertigo in the Community. Arch Intern Med. 2008;168(19):2118-2124.

4. Stoll W, Matz DR, Most E. Schwindel und Gleichgewichtsstörungen.Georg Thieme Verlag. Stuttgart -New York 1986: 33-284.

5. Aguilar C, Diagnóstico y tratamiento de las dislipidemias, posición de la SMNE, Revista de Endocrinología y nutrición. 2004; (12) 1: 8-39.

6. Koch F. Dislipidemias en el 2005 conceptos actuales. Revista F M Universidad de Tucuman, Argentina. 2005; 6 (1) :8-12.

7. Scott M, Becker D,Luther T, Richard S, Margo A, Hunninghake D, et al. Third Report of the National Cholesterol Education Program (NCEP) Expert Panel on Detection, Evaluation, and Treatment of High Blood Cholesterol in Adults (ATP III) Final Report .Circulation. 2002 106: 3143.

8. Fife TD. Episodic vertigo In: Rakel and Bope. Conn's Current Therapy 2009. Philadelphia: Elsevier; 2009. p. 204-210.

9. Milionis HJ, Mittari V, Exarchakos G, Kalaitzidis R, Skevas AT, Elisaf MS. Lipoprotein (a) and acute-phase response in patients with vestibular neuronitis.; EJCI. 2003; (33)12:104550.

10. Tatsuhito O,Masaharu U,Yutaka N. A study of tinnitus vertigo patients with hyperlipidemia. Otologia Fukuoka J. 2002; 48:313-318.

11. Kazmierczak H, Doroszewska G. Metabolic disorders in vertigo, tinnitus, and hearing loss.Int Tinnitus J. 2001; (7)1:54-8.

12. Pulec JL, Pulec MB, Mendoza I. Progressive sensorineural hearing loss, subjective tinnitus and vertigo caused by elevated blood lipids. Ear Nose Throat J. 1998; (77) 3:224.

13. Rybak L. Metabolic disorders of the vestibular system. Otolaryngol Head Neck Surg. 1995; (112)1:128-32.

14. Neuhauser HK, Von Brevern M, Radtke A, Lezius F, Feldmann M, Ziese T,Lempert T. Epidemiology of vestibular vertigo: a neurotologic survey of the general population. Neurology. 2005; 65: 898-904.

15. Colledge N. Evaluation of investigations to diagnose the cause of dizziness in elderly people: a community based controlled study. BJM. 1996; 313:788.

16. Baloh R. The dizzy patient. Postgrad Med. 1999; (105) 2:161.

17. Knox GW, McPherson A. Enfermedad de Meniere. Am Fam Physician 1997; (4) 55 :1185.

18. Brandt T, Dieterich M, Strupp M. Vertigo and dizziness: common complaints. London: Springer; 2005. p. 148.

19. Fife TD. Episodic Vertigo. In: Rakel \& Bope: Conn's Current Therapy 2009. Philadelphia: Elsevier; 2009. pp 204-210.

20. Derebery MJ. The diagnosis and treatment of dizziness. Med Clin North Am. 1999; 83:163176.

21. Espinosa JM, Cervera J. El paciente con vértigo en urgencias, Rev Med Univ Navarra. 2003; (47) 4:77-81.

22. Nicki R, Robin M, Barr-Hamilton S, Lewis J, Robin J, Sellar A. Evaluation of investigations to diagnose the cause of dizziness in elderly people: a community based controlled study. BMJ. 1996; 313:788-92.

23. Kazmierczak H, Doroszewska G. Metabolic disorders in vertigo, tinnitus, and hearing loss. Int Tinnitus J. 2001; (7)1:54-8.

24. Lin ZM, Young YH. Investigating the causes of vertigo in breast cancer survivors. Eur Arch Otorhinolaryngol. 2005; 262 (5):432-6.

25. Brown J. A systematic approach to the dizzy patient. Neurologic Clinics. 1990; 8:209-224.

26. Halevy A. Naveh E. Measuring and Reducing the National Cost of non-Quality. Total Quality Management. 2000; 11:1095-1110. 